\title{
The Development of Electrochemical Aptasensor Based on DNA Aptamers Modified by Redox Markers for Detection of Leukemia Jurkat Cells ${ }^{\dagger}$
}

\author{
Cyril Slabý ${ }^{1}$, Lenka Bábelová ${ }^{1,2}$ and Tibor Hianik ${ }^{1, *}$ \\ 1 Faculty of Mathematics, Physics and Informatics, Comenius University, Mlynská dolina F1, \\ 84248 Bratislava, Slovakia; slaby.cyril@gmail.com \\ 2 Institute of Animal Biochemistry and Genetics, Center of Biosciences SAS, Dúbravská cesta 9, \\ 84005 Bratislava, Slovakia; babelova@me.com \\ * Correspondence: tibor.hianik@fmph.uniba.sk \\ + Presented at the 1st International Electronic Conference on Biosensors, 2-17 November 2020; \\ Available online: https://iecb2020.sciforum.net/
}

Received: date; Accepted: date; Published: date

\begin{abstract}
Oncological diseases belong to the most serious illnesses with high mortality. The most common cancer in children is acute lymphoblastic leukemia (ALL). It is important to develop diagnostic methods that will be able to detect this disease in early stage. One of the possible options can be non-invasive diagnostics using the biosensors based on nucleic acid aptamers. Aptamer recognizes the surface markers on the membrane of cancer cells with the high binding affinity. Biosensors based on aptamers with redox markers are among the most sensitive experimental tools of this type. We developed and optimized the redox-labeled electrochemical aptasensors for the detection of Jurkat leukemia cells. The aptamers specific to the protein tyrosine kinase 7 (PTK7), which is important membrane protein cancer marker that is overexpressed in Jurkat cells were used. We compared the sensitivity of aptasensors for aptamers modified either by methylene blue (MB) and ferrocene carboxylic acid $(\mathrm{Fc})$, respectively. Both aptasensors were tested in the presence of Jurkat cells at concentration range 50-5000 cells/mL using differential pulse voltammetry. In both cases the comparable sensitivity was obtained with limit of detection: $37 \pm 6$ cells $/ \mathrm{mL}$ for Fc labeled aptamers and $38 \pm 8$ cells $/ \mathrm{mL}$ for MB labelled aptamers based on 3.3S/N (signal to noise) rule. The interaction of the sensing surface with control U266 cells was less significant.
\end{abstract}

Keywords: acute lymphoblastic leukemia; aptasensor; electrochemistry; Sgc8c aptamer; methylene blue; ferrocene carboxylic acid

\section{Introduction}

The noninvasive early diagnosis of a cancer and monitoring of its progress may increase the chance of successful treatment. Cancer of blood including leukemia, lymphoma, and myeloma is one of the most common types of cancers [1]. Acute lymphoblastic leukemia (ALL) is an aggressive form of leukemia that originates in a single B- or T-lymphocyte progenitor. The disease is most common in children but can occur at any age. Currently, $80 \%$ of children and $35 \%$ of adults can expect longterm leukemia-free survival, and probable cure, following intensive therapy on contemporary protocols.

The standard and representative assays for the detection of PTK7 protein are real-time quantitative PCR, flow cytometry, and ELISA kit [2]. These methods are, however, time-consuming, multi-steps and expensive tests. The flow cytometry requires large number of cell samples. ELISA is an important immunoassay to detect various disease protein biomarkers and pathogens with the 
LOD down to subnano gram per $\mathrm{mL}$ [3]. On the other hand, it suffers from the false positive results by the cross-reactivity in antigen-antibody recognition.

Therefore, the development of innovative and effective technologies is of great interest for leukemia diagnosis. Nanotechnology, especially biosensors, involves a multidisciplinary research field such as molecular engineering, biology, chemistry and physics [4]. In this study, we report new approach for detection leukemia cells. We explored the DNA aptamers modified by redox probes to design the electrochemical sensor. The principal signaling mechanism in this class of sensors relates to conformation and/or flexibility changes in the electrode bound aptamers [5]. Within these efforts to develop aptamers of improved binding properties and enhanced functionalities, the chemical modification of the aptamers attracted scientific challenge. These included the chemical modification of aptamers with redox labels as an amplification strategy for electrochemical sensors [6]. The DNA aptamer specific to cancer marker at the membrane of Jurkat leukemic cells can be modified at the $5^{\prime}$ end by biotin or SH group for immobilization at the electrode and at 3'-end, with a redox probe such as methylene blue $(\mathrm{MB})$ or ferrocene carboxylic acid $(\mathrm{Fc})$. The convenience of reversible electrochemical behaviour and standard conjugation chemistry makes both molecules ideal candidates for use as signalling moieties.

In this study, we used sgc8c DNA aptamer that can recognize the PTK7, a transmembrane receptor, highly expressed on Jurkat leukemic cells. The screening of the PTK7 biomarker is crucial for point-of-care diagnosis. The biomarkers are involved in many steps of cancer management: they can be used not only for diagnosis, but also for assessing risk and prognosis or monitoring therapeutic efficiency. In this article, we described detection of Jurkat cells by two redox markers modified aptamers and compared sensitivity of detection with so far published papers.

\section{Materials and Methods}

Electrode preparation: In brief, prior to sensor preparation, gold disk electrodes $(2 \mathrm{~mm}$ diameter, CHI 101, CH Instruments, USA) were cleaned mechanically (by polishing with 0.3 and 1.0 $\mu \mathrm{m}$ alumina oxide slurries on a nylon support), then cleaned by ultrasound ( $\mathrm{f}=35 \mathrm{kHz}$ ) and by electrochemical method (by cyclic voltammetry through successive 20 scans in $0.5 \mathrm{M}$ sulfuric acid) as previously described [7].

Preparation of aptamer-based sensor: DNA aptasensors were prepared using established procedures. Thiolated aptamer sgc8c modified by methylene blue as redox marker: $\mathrm{SH}$-sgc8c-MB; $5^{\prime}-$ SH-C6SS-ATC TAA CTG CTG CGC CGC CGG GAA AAT ACT GTA CGG TTA GA-MB-3' (Biosearch Technologies, Denmark) was dissolved in nuclease free water. The cleaned gold electrodes were immersed in $100 \mu \mathrm{L}$ of $1 \mu \mathrm{M}$ solution of SH-sgc8c-MB aptamer in $10 \mathrm{mM}$ PBS (pH 7.4) for $16 \mathrm{~h}$. The immobilization of the aptamers took place at absence of light, because MB is light sensitive. After 16 $\mathrm{h}$, the electrodes were rinsed with MilliQ water and immersed in $100 \mu \mathrm{L}$ of $7 \mathrm{mM}$ solution of 6mercapto-1-hexanol (MCH, Sigma Aldrich, Germany) for $1 \mathrm{~h}$. The electrode surface was "backfilled" with $\mathrm{MCH}$ to form a continuous, mixed, self-assembled monolayer. The incubation of the electrodes in the $\mathrm{MCH}$ also took place without accesses the light. After $1 \mathrm{~h}$ of incubation in $\mathrm{MCH}$, the electrodes were rinsed in MilliQ water for 5-10 min to remove non-specifically bounded $\mathrm{MCH}$. Such an electrode was ready for the measurements.

For aptamer modification by Fc the biotinylated aptamer sgc8c: Biotin-sgc8c-NH2; 5'-BiotinATC TAA CTG CTG CGC CGC GAA AAT ACT GTA CGG TTA GA-NH $2-3$ ' (Eurogentec, Belgium) modified by amino group at 3'was dissolved in TE buffer (10 mM TRIS + 1 mM EDTA, pH 8). The clean electrodes were immersed in $200 \mu \mathrm{L}$ of $125 \mu \mathrm{g} / \mathrm{mL}$ neutravidin (NA, Thermo Fisher Scientific, USA) in MilliQ water. The incubation of electrodes took place for 20 min without access the light, as NA is light sensitive. To remove excess of an unbound NA, we placed the electrodes in $500 \mu \mathrm{L}$ of MilliQ water for $30 \mathrm{~min}$. Then small amount (approx. $10 \mu \mathrm{L}$ ) of the $1 \mu \mathrm{M}$ aptamer dissolved in PBS has been dropped at the surface of NA-modified electrode, covered by Eppendorf tube and incubated during $30 \mathrm{~min}$. Then the surface of the electrode was rinsed with deionized water. The modification of aptamers by $\mathrm{Fc}$ has been performed as follows. First the carboxylic groups in $20 \mathrm{nM}$ ferrocenecarboxylic acid $(\mathrm{Fc})$ have been activated in a mixture of $200 \mu \mathrm{L}$ of $5 \mathrm{mM}$ NHS and $1 \mathrm{mM}$ EDC for $2 \mathrm{~h}$. 
The Fc has been then added at the surface of the aptasensor for $30 \mathrm{~min}$ and then rinsed by deionized water. Such a sensor has been ready for the experiments.

Electrochemical studies: Electrochemical measurements were performed on $\mu$ Autolab III (Eco Chemie, The Netherlands) controlled by GPES (General Purpose Electrochemical System). We used differential pulse voltammetry (DPV) as detection method. In this method, the potential is applied in pulses [8]. Current sampling takes place twice during each potential pulse, before the start of the pulse and then at the end of the pulse. Each pulse creates one measured point according to the relation:

$$
I_{n 2}-I_{n 1}=\Delta I_{n, d p}
$$

where $n$ is the order of the pulse. The resulting voltammogram consists of discrete values $\Delta I_{n, d p}$ displayed as a function of potential [9]. The following parameters were applied in DPV measurements: scan rate $0.25 \mathrm{mV} / \mathrm{s}$, pretreatment $120 \mathrm{~s}$.

In experiments we used Jurkat cells (PTK7-positive human suspension T-lymphoblasts of ALL). For non-specific interactions, we selected control U266 cells (PTK7-negative B-lymphocytes). The cell lines were supplied from the Cancer Research Institute of the Slovak Academy of Sciences.

\section{Results and Discussion}

\subsection{Optimization of Measuring Conditions (Incubation Time, Stability, Aptamer Concentration)}

In the present study, we have examined two important types of redox-labelled sgc8c aptamers consisting of $\mathrm{MB}$ or Fc. The redox-active units are responsible for electron production and signal amplification in an electrochemical biosensor. The signal changes are influenced by the binding properties of the Jurkat leukemic and U266 control cells to the aptamer binding site (Scheme 1).

1

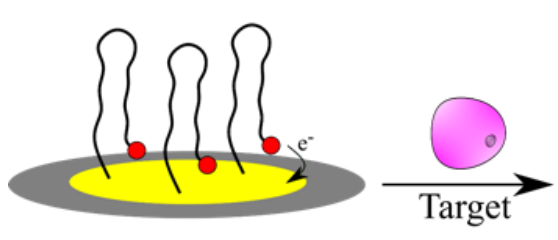

2

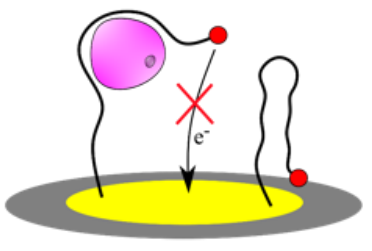

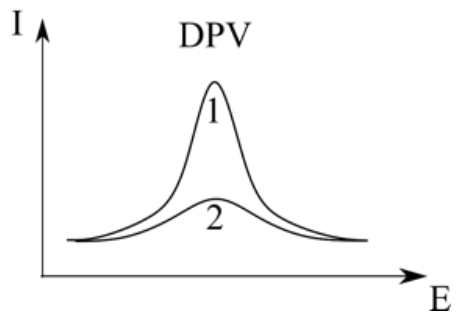

Scheme 1. The scheme of electrochemical aptasensor. Signal off: binding reduces the current from the redox tag (red circle). Aptasensor before binding of target (part 1) and after binding the cells (purple object, part 2). DPV current changes correspond to binding phenomenon.

For each of these modified aptamers, we determined the incubation time, optimal potential window and aptamer concentration. A DNA aptasensor containing PTK7-binding aptamer was thiolated, conjugated with redox tag, and immobilized on a gold electrode by self-assembly. The reaction time is an important parameter for target cell capture and the specific recognition of the cells and aptamer. The incubation time of $60 \mathrm{~min}$ was chosen as an optimal for detection of leukemic or control cells. In order to verify the stability of the prepared electrode, consecutive voltammograms were obtained in the presence of only of the working buffer at a fixed scan rate. The electrochemical responses were interrogated using differential pulse voltammetry. We have characterized the potential window ( -0.3 to $-0.1 \mathrm{~V}$ vs. $\mathrm{Ag} / \mathrm{AgCl}$ in $10 \mathrm{mM}$ PBS for MB-APT and -0.1 to $+0.6 \mathrm{~V}$ vs. $\mathrm{Ag} / \mathrm{AgCl}$ in $100 \mathrm{mM}$ Hepes for Fc-APT) over which DPV peak current was stable for $3 \mathrm{~h}$. To evaluate the sensitivity of the prepared aptasensor, experiments using four different concentrations of Sgc8c aptamer $(0.1,1,3$ and $5 \mu \mathrm{M})$ in the presence of 1000 Jurkat cells/mL were performed. The resulting electrochemical oxidation peak current was measured. When the APT concentration increased, the DPV signal decreased probably due to enhanced hindering of electron transfer reaction of redox probe on the sensor surface. We found out the optimal concentration of $1 \mu \mathrm{M}$ of sgc8c. 


\subsection{Sensitivity of the Aptasensor}

After the preparation of aptasensor, DPV based electrochemical detection was carried out using the optimal parameters. Figure 1 shows the DPV curves obtained in blank working buffer $(0$ cells $/ \mathrm{mL})$ and then in presence of leukemic cells. In physiological conditions, MB is reduced in leucomethylene blue, the process involves $2 \mathrm{e}^{-}$and $1 \mathrm{H}^{+}[10]$. Upon the oxidation of $\mathrm{MB}$ tagged on aptamers, the height of the resulting peak wave form was recorded. The interaction of PTK7 receptor on the membrane of leukemic cells with the aptasensor was proved by three different concentrations 50, $5 \times 10^{2}, 5 \times 10^{3}$ Jurkat cells/mL. After one hour of incubation of the aptasensor with the cell suspension the electrochemical properties were efficiently detected by DPV (Figure 1a). We could observe decreasing peak current with the increasing concentration of Jurkat cells. The main reason of signal decreasing is the formation of Jurkat cells-Sgc8c complexes, which inhibited the electron transfer [11]. In another aptasensor design, the aptamers modified by Fc redox marker was tested. In the absence of the leukemic cell target, the immobilized DNA probe remained relatively unfolded, allowing the electron transfer from the Fc label to the electrode surface. Figure $1 \mathrm{~b}$ displays the DPV curves of the biosensor in the presence of Jurkat cells with different concentrations. Fc undergoes a reversible, one-electron oxidation. We can recognize differences between the signal changes caused by the MB and Fcmodified aptamer after cells binding.

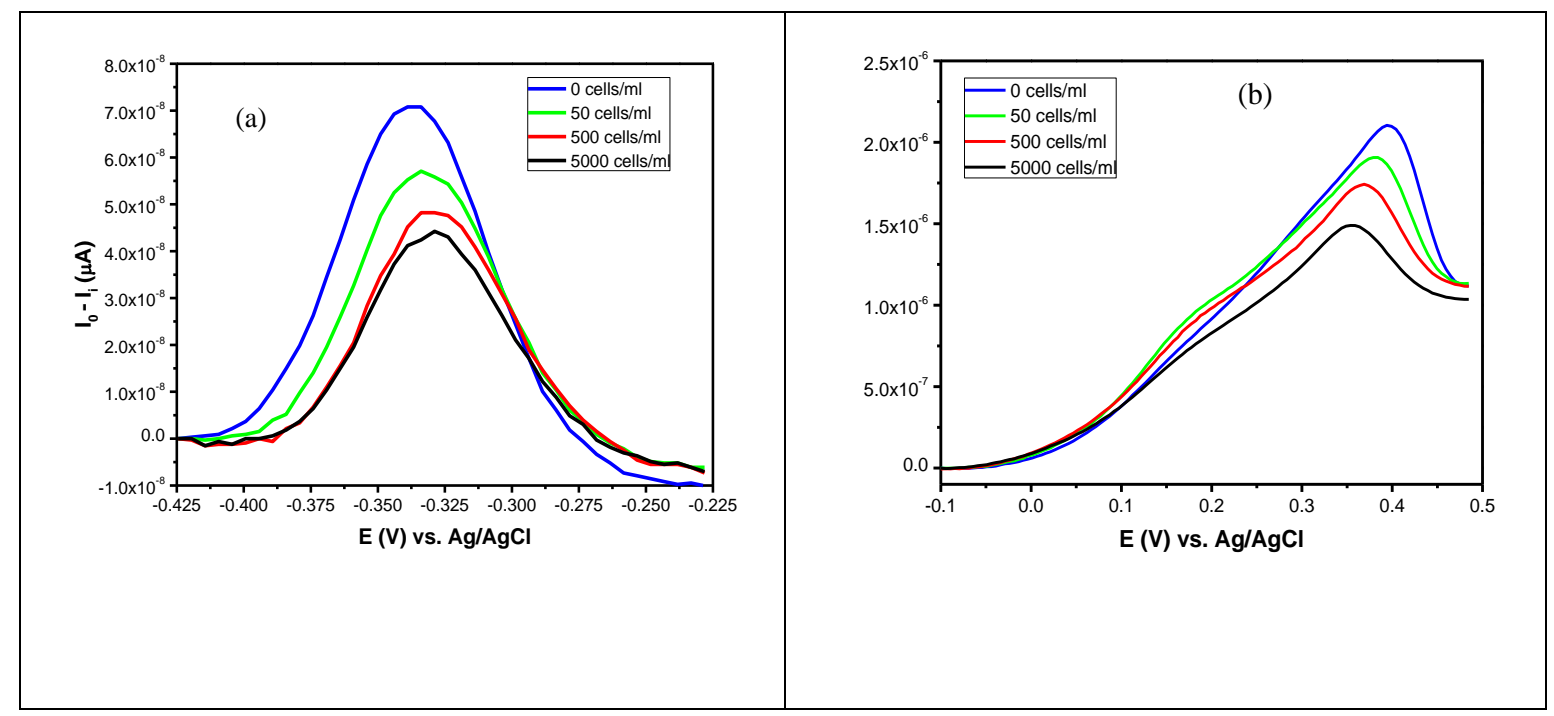

Figure 1. Differential pulse voltammograms of methylene blue (a) and ferrocene carboxylic acid (b) modified aptamer at different Jurkat cell concentrations in working buffer at pH 7.4. DPV has been baseline corrected.

Target detection is performed by measuring changes in voltammetric peak current. In our research, we tested Jurkat (T-cell, acute lymphoblastic leukemia) and the control U266 (Blymphocytes, a PTK7 negative cell line) cell lines. The aptasensors were incubated with various concentration of 50, $5 \times 10^{2}, 5 \times 10^{3}$ cells $/ \mathrm{mL}$ in $8 \mathrm{~mL}$ Teflon electrochemical cell. Each concentration was incubated for $60 \mathrm{~min}$. After one-hour binding process between cells and sgc8c aptamer, the sensor was rinsed with MilliQ water and DPV response was measured. Typically defined as relative current changes and plotted against concentration of Jurkat cells to construct a calibration curve. Each point on the calibration curve corresponds to the mean value obtained from three independent measurements (Figure 2). 


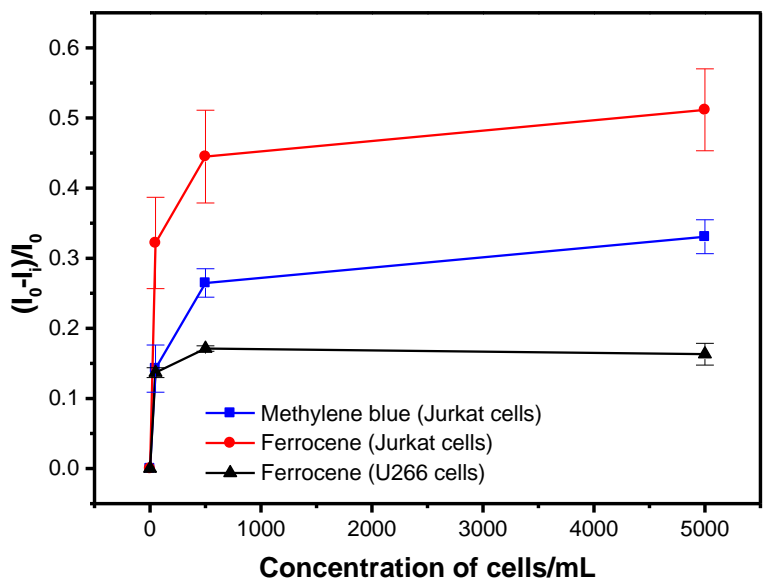

Figure 2. The relative changes of the peak current intensity of the two types electrochemical aptasensors vs. cell concentration. The error bars were obtained from three experiments. Io is peak current prior addition and $\mathrm{I}_{\mathrm{i}}$ after addition of the cells in a respective concentration.

Figure 2 shows rapid changes of the peak current at relatively small cell concentration with saturation at around $500 \mathrm{cells} / \mathrm{mL}$. The signal produced by Fc has been approx. 1.6 times higher. Based on the 3.3S/N (signal to noise) rule the limit of detection (LOD) has been determined for Fc and MB based aptasensor: $37 \pm 6$ cells $/ \mathrm{mL}$ and $38 \pm 8$ cells $/ \mathrm{mL}$, respectively. These values are similar.

The obtained detection limits are rather low and can be compared with other studies that used electrochemical and acoustic methods of detection. As it can be seen from Table 1, the results that have been obtained by Fc and MB labelled aptamers presented in our work belongs among the most sensitive detection assay. At the same time, the sensor preparation as well as detection method is rather simple and effective. In addition, recently have been published papers that shows also high sensitivity for diagnosis of leukemia by detection specific antigens. Mazloum-Ardakani et al. [19] published work, where used carcinoembryonic antigen (CEA) as a biomarker for ALL detection. They constructed disposable cancer-screening package included a DNA sensor and aptasensor consisting of nanostructures like carbon quantum dots and gold nanoparticles. Prepared package of biosensors was characterized using the electrochemical methods. The LOD of $0.26 \mathrm{pg} / \mathrm{mL}$ for the CEA antigen was comparable vs. the Cell Biolabs ELISA kit. Sensitive and specific detection in the solution and directly on the surface of live CCRF-CEM cells with overexpressed PTK7 demonstrated Li and coworkers using surface-enhanced Raman scattering [2]. They developed the synergistic combination of the conformational alteration of the anglerfish aptamer triggered by the recognition of the PTK7 and Exo III enzyme-assisted nucleic acid amplification. The limit of detection was better than ELISA by nearly 5 orders of magnitude. Grechkin et al. developed luminescent nanoparticles with sgc8 aptamer and detected leukemia cells using fluorescence microscopy [20]. They confirmed sgc8 binding with CCRF-CEM and Jurkat cell lines. This approach allowed distinguishing the leukemia cells by fluorescence microscopy. 
Table 1. Comparison of the properties of electrochemical and acoustics aptasensors for detection cancer cells.

\begin{tabular}{|c|c|c|c|c|c|c|}
\hline Cell Line & Apta-Mer & Immobilization Method & Met-Hod & $\begin{array}{c}\text { Linear Range } \\
\text { Cells } / \mathrm{mL}\end{array}$ & $\begin{array}{c}\text { LOD } \\
\text { Cells/mL }\end{array}$ & Ref. \\
\hline $\begin{array}{l}\text { CCRF- } \\
\text { CEM }\end{array}$ & $\mathrm{Sgc} 8 \mathrm{c}$ & $\begin{array}{l}\text { Sandwich immo-bilization PEDOT-Aunano- } \\
\text { sgc8c aptamer-cells and catalytic labaled sgc8c } \\
\text { aptamer (MWCNTs-Pdnano/PTCA/aptamer) }\end{array}$ & EIS,CV & $1-5 \times 10^{5}$ & 8 & {$[12]$} \\
\hline DLD-1 & $\begin{array}{l}\text { MUC-1- } \\
\text { aptamer }\end{array}$ & $\begin{array}{l}\text { MUC-1 aptamer bound on carbon nanospheres } \\
\text { (CNSs) }\end{array}$ & EIS,CV & $1.25 \times 10^{2}-1.25 \times 10^{6}$ & 40 & [13] \\
\hline CT26 & $\begin{array}{l}\text { SBA-15-pr- } \\
\mathrm{NH}_{2}\end{array}$ & $\begin{array}{l}\text { Sandwich architecture: aptamer-cell-aptamer: } \\
\text { SBA-15-pr-NH} 2 \text { and AuNPS modified electrode }\end{array}$ & EIS, CV & $\begin{array}{c}10^{-1} \times 10^{5} \text { and } 1 \times 10^{5}-6 \times \\
10^{6}, \mathrm{CV} \text { and EIS }\end{array}$ & 2 & {$[14]$} \\
\hline $\begin{array}{l}\text { CCRF- } \\
\text { CEM }\end{array}$ & Sgc8c & Self assembly of thiol-terminated aptamer & EIS,CV & $1 \times 10^{4}$ to $1 \times 10^{7}$ & $6 \times 10^{3}$ & {$[15]$} \\
\hline $\begin{array}{l}\text { HepG2, } \\
\text { HeLA }\end{array}$ & $\begin{array}{l}\text { Aptamer to } \\
\text { A549 cells }\end{array}$ & EDC-NHS-aptamer & EIS & - & 163.7 & {$[16]$} \\
\hline Jurkat & Sgc8c & $\begin{array}{l}\text { Self assembly of thiol-terminated aptamer }+ \\
\mathrm{MCH}\end{array}$ & EIS & $50-500 \times 10^{3}$ & $105 \pm 10$ & [7] \\
\hline $\begin{array}{l}\text { CCRF- } \\
\text { CEM }\end{array}$ & Sgc8c & $\begin{array}{l}\text { Self assembly of thiol-terminated aptamer }+ \\
\mathrm{MCH}\end{array}$ & QCM & $2 \times 10^{3}-1 \times 10^{5}$ & 1160 & {$[17]$} \\
\hline Jurkat & Sgc8c & $\begin{array}{l}\text { Self assembly of thiol-terminated aptamer }+ \\
\mathrm{MCH}\end{array}$ & QCM & $50-500 \times 10^{3}$ & $463 \pm 50$ & {$[18]$} \\
\hline Jurkat & $\operatorname{Sgc} 8 \mathrm{c}$ & $\begin{array}{l}\text { Self assembly of thiolated aptamers at gold } \\
\text { surface. Aptamers modified by MB redox label }\end{array}$ & DPV & $50-500$ & $38 \pm 8$ & This work \\
\hline Jurkat & Sgc8c & $\begin{array}{l}\text { Biotinylated aptamers modified by ferrocene and } \\
\text { immobilized at gold surface }\end{array}$ & DPV & $50-500$ & $37 \pm 6$ & This work \\
\hline
\end{tabular}




\section{Conclusions}

We have developed a novel electrochemical sensing platform for the detection of leukemia cells using sgc8c DNA aptamers specific to cancer biomarker PTK7. Using rather simple aptasensor preparation either by chemisorption of thiolated aptamers on a gold surface or biotinylated aptamers on a gold surface with chemisorbed neutravidin and using MB or Fc labelled aptamers, it was possible to detect Jurkat leukemic cells with rather high sensitivity and selectivity $(37 \pm 6$ cells $/ \mathrm{mL}$ for Fc and $38 \pm 8$ cells/mL for MB labelled aptamers, respectively). The DPV method used allowed straightforward detection of the cancer cells at the surface of the sensor. The proposed approach can be perspective for further practical works toward diagnostic of leukemia at early stage.

Author Contributions: conceptualization, T.H.; methodology, T.H.; investigation, C.S. and L.B.; formal analysis, C.S. and L.B.; resources, T.H.; writing-original draft preparation, C.S. and L.B.; writing-review and editing, T.H.; supervision, T.H.; project administration, T.H.; funding acquisition, T.H. All authors have read and agreed to the published version of the manuscript.

Funding: The research was financially supported by Science Agency VEGA, project No. 1/0419/20.

Acknowledgments: We acknowledge Jozef Bizík and Monika Buríková from the Cancer Research Institute, Biomedical Research Center of the Slovak Academy of Sciences for cell cultures used in the experiments.

Conflicts of Interest: The authors declare no conflict of interest.

\section{References}

1. Liu, G.; Mao, X.; Phillips, J.A.; Xu, H.; Tan, W.; Zeng, L. Aptamer-nanoparticle strip biosensor for sensitive detection of cancer cells. Anal. Chem. 2009, 81, 10013-10018.

2. Li, Y.; Fang, Q.; Miao, X.; Zhang, X.; Zhao, Y.; Yan, J.; Zhang, Y.; Wu, R.; Nie, B.; Hirtz, M.; et al. Aptamer conformation-cooperated enzyme-assisted surface-enhanced Raman scattering enabling ultrasensitive detection of cell surface protein biomarkers in blood samples. ACS Sens. 2019, 4, 2605-2614.

3. Lin, Y.; Zhang, L.-H.; Wang, X.-H.; Xing, X.-F.; Cheng, X.-J.; Dong, B.; Hu, Y.; Du, H.; Li, Y.-A.; Zhu, Y.-B.; et al. PTK7 as a novel marker for favorable gastric cancer patient survival. J. Surg. Oncol. 2012, 106, 880886.

4. Avelino, K.Y.P.S.; Silva, R.R.; da Silva Junior, A.G.; Oliveira, M.D.L.; Andrade, C.A.S. Smart applications of bionanosensors for BCR/ABL fusion gene detection in leukemia. J. King Saud Univ. Sci. 2017, 29, 413-423.

5. Catanante, G.; Mishra, R.K.; Hayat, A.; Marty, J.-L. Sensitive analytical performance of folding based biosensor using methylene blue tagged aptamers. Talanta 2016, 153, 138-144.

6. Biniuri, Y.; Luo, G.-F.; Fadeev, M.; Wulf, V.; Willner, I. Redox-switchable binding properties of the ATPaptamer. J.Am. Chem. Soc. 2019, 141, 15567-15576.

7. Bábelová, L.; Sohová, M.E.; Poturnayová, A.; Buríková, M.; Bizík, J.; Hianik, T. Label-free electrochemical aptasensor for Jurkat cells detection as a potential diagnostic tool for leukamia. Electroanalysis 2018, 30, 1487-1495

8. Girault, H.H. Analytical and Physical Electrochemistry, 1st ed.; EPFL Press: Lausanne, Switzerland, 2004; p. 315.

9. Scholz, F. Voltammetric techniques of analysis: The essentials. ChemTexts 2015, 1, 17.

10. Barou, E.; Bouvet, M.; Heintz, O.; Meunier-Prest, R.; Electrochemistry of methylene blue at an alkanethiol modified electrode. Electrochim. Acta 2012, 75, 387-392.

11. Chiorcea-Paquim, A.-M.; Oliveira-Brett, A.M. Guanine quadruplex electrochemical aptasensors. Chemosensors 2016, 4, 13.

12. Tabrizi, M.A.; Shamsipur, M.; Saber, R.; Sarkar, S. Flow injection amperometric sandwich-type aptasensor for the determination of human leukemic lymphoblast cancer cells using MWCNTs-Pdnano/PTCA/aptamer as labeled aptamer for the signal amplification. Anal. Chim. Acta 2017, 985, 61-68.

13. Cao, H.; Ye, D.; Zhao, Q.; Luo, J.; Zhanga, S.; Kong, J. A novel aptasensor based on MUC-1 conjugated CNSs for ultrasensitive detection of tumor cells. Analyst 2014,139, 4917-4923. 
14. Hashkavayi, A.B.; Raoof, J.B.; Ojani, R.; Kavoosian, S. Ultrasensitive electrochemical aptasensor based on sandwich architecture for selective label-free detection of colorectal cancer (CT26) cells. Biosens. Bioelectr. 2016, 92, 630-637.

15. Pan, C.F.; Guo, M.L.; Nie, Z.; Xiao, X.L.; Yao, S.Z. Aptamer-based electrochemical sensor for label-free recognition and detection of cancer cells. Electroanalysis 2009, 21, 1321-1326.

16. Kara, P.; Erzurumlu, Y.; Kirmizibayrak, P.B.; Ozsoz, M. Electrochemical aptasensor design for label free cytosensing of humannon-small cell lung cancer. J. Electroanal.Chem. 2016, 775, 337-341.

17. Shan, W.; Pan, Y.; Fang, H.; Guo, M.; Nie, Z.; Huang, Y.; Yao, S. An aptamer-based quartz crystal microbalance biosensor for sensitive and selective detection of leukemia cells using silver-enhanced gold nanoparticle label. Talanta 2014, 126, 130-135.

18. Poturnayová, A.; Buríková, M.; Bízik, J.; Hianik, T. DNA aptamers in the detection of leukemia cells by the thickness shear mode acoustics method. ChemPhysChem 2019, 20, 545-554.

19. Mazloum-Ardakani, M.; Barazesh, B.; Moshtaghiun, S.M. A distinguished cancer-screening package containing a DNA sensor and an aptasensor for early and certain detection of acute lymphoblastic leukemia. Clin. Chim. Acta 2019, 497, 41-47.

20. Grechkin, Y.A.; Grechkina, S.L.; Zaripov, E.A.; Fedorenko, S.V.; Mustafina, A.R.; Berezovski, M.V. Aptamer-conjugated $\mathrm{Tb}(\mathrm{III})$-doped silica nanoparticles for luminescent detection of leukemia cells. Biomedicines 2020, 8, 14 .

Publisher's Note: MDPI stays neutral with regard to jurisdictional claims in published maps and institutional affiliations.

(C) 2020 by the authors. Submitted for possible open access publication under the terms and conditions of the Creative Commons Attribution (CC BY) license (http://creativecommons.org/licenses/by/4.0/). 